\title{
Waste as a Resource for Avifauna: Review and Survey of the Avifaunal Composition in and around Waste Dumping Sites and Sewage Water Collection Sites (India)
}

Satya Prakash Mehra*, Sarita Mehra, Mohib Uddin, Vikas Verma, Hrishika Sharma, Tehlu Singh, Gurpreet Kaur, Tasso Rimung and Himmat Ram Kumhar

Rajputana Society of Natural History, Village Ramnagar, PO Malah, Bharatpur, Rajasthan, India

\begin{abstract}
Urbanization has lead to the challenge of waste disposal. The dumping sites are affecting the natural habitats in and around urban areas. The most eye catching group of animals, the birds had used these modified habitats. The reconnaissance surveys were conducted for eleven sites of the seven municipal areas Rajasthan and Punjab states of India to assess the avifaunal composition. The observations of bird species using modified habitats in form of solid and liquid (effluent/ sewage) waste sites were assessed. The dumping site at Mount Abu (Sirohi, Rajasthan) was no more in existence. Since authors were involved in studies since last two decades, therefore, past records were also included for such sites.

It was observed that such sites of waste collection (solid and liquid) were harboring 100 species of birds with three additional species in past, accounting 103 bird species belonging to 37 families in 11 orders. Terrestrial species accounted 53 whereas wetland bird species were 37 species and 11 species were wetland dependent. Around 58 species were resident, 18 migrants and 27 species resident with local movements. Thirteen species of global interest were recorded from the sites. Three of these species were under critically endangered and were the past records. Two endangered species, one vulnerable species and seven near threatened species were recorded from the investigation sites. Sites of Udaipur and Bharatpur were having the maximum diversity of birds.

Besides scavenger and raptors species, egrets and passerines were of common occurrence. It was observed that the sites were mainly used for the feeding purposes and if the surrounding habitats were used for the other life cycle processes by the birds. The dumping sites with the organic (biodegradable and animal) wastes could be prepared and further modified as per the nature's rule to develop the birding sites for the species of global interest. The animal waste management through reviving bio-disposal mechanism through scavenger birds could be ideal model for revenue generation through birding.
\end{abstract}

Keywords: Human; Nature; Environment

\section{Introduction}

\section{Importance of the theme}

Twenty-first century can be characterized by tremendous growth of urban areas along with associated process of globalization and unification of urban environments. Despite of the fact that cities occupy just $2 \%$ of the Earth's surface, their inhabitants use $75 \%$ of the planet's natural resources $[1,2]$. Due to changes in the habitats and the direct human disturbances, the urban development processes affect avifauna by various means, which might be positive or negative [3]. However, some bird species can thrive in human-modified landscapes, if the habitats retain ecologically important features $[1,2,4]$. There are ample of studies on relation of the landscapes with the abundance of animal populations especially where the anthropogenic activities are affecting the natural characteristics of the habitats [2,5-7] and owing to the home ranges this is the case particularly for birds [4]. The birds are the most eye catching group of animals among all at any site or habitats whether wild or modified [8].

India is facing a challenge of the ever increasing urban population due to lack of available services and resources resulting into the heap of garbage dump and sewer waste water [9]. The unpleasant odor of the decomposing wastes infuses everywhere. However, the garbage dumping and the waste water sites are being used by diverse species of invertebrates and vertebrates $[10,11]$. The surveys were undertaken by the authors to assess the avifaunal species of such sites from selected urban lands of Rajasthan and Punjab. The paper enlisted the species of birds recorded from investigation sites.

\section{Materials and Methods}

\section{Study area}

Seven urban areas from two states of India, four from Rajasthan and three from Punjab were surveyed by the authors with the prime objective to assess the avifaunal composition of all the sites of dumping grounds as well as waste water collection irrespective of their status identified by the respective municipal bodies. The selection of urban areas was based on the authors working areas. The urban spaces included municipal areas of Udaipur, Mount Abu, Bharatpur and Kota from Rajasthan and Nawanshahr (Shaheed Bhagat Singh Nagar), Moga and Ludhiana from Punjab (Figure 1). Six of them are district headquarters whereas Mount Abu falls in Sirohi District of Rajasthan.

Seven urban areas had eleven sites under two broad types of habitats - seven terrestrial habitats in form of dumping grounds $(\mathrm{T})$

*Corresponding author: Mehra SP, Rajputana's Shakuntalam, Village Ramnagar, PO Malah, Bharatpur 321001 Rajasthan, India, Tel: +91 9414165690; E-mail: drspmehra@yahoo.com

Received July 03, 2017; Accepted July 10, 2017 ; Published July 17, 2017

Citation: Mehra SP, Mehra S, Uddin M, Verma V, Sharma H, et al. (2017) Waste as a Resource for Avifauna: Review and Survey of the Avifaunal Composition in and around Waste Dumping Sites and Sewage Water Collection Sites (India). Int J Waste Resour 7: 289. doi: 10.4172/2252-5211.1000289

Copyright: $\odot 2017$ Mehra SP, et al. This is an open-access article distributed under the terms of the Creative Commons Attribution License, which permits unrestricted use, distribution, and reproduction in any medium, provided the original author and source are credited. 
Citation: Mehra SP, Mehra S, Uddin M, Verma V, Sharma H, et al. (2017) Waste as a Resource for Avifauna: Review and Survey of the Avifaunal Composition in and around Waste Dumping Sites and Sewage Water Collection Sites (India). Int J Waste Resour 7: 289. doi: 10.4172/22525211.1000289
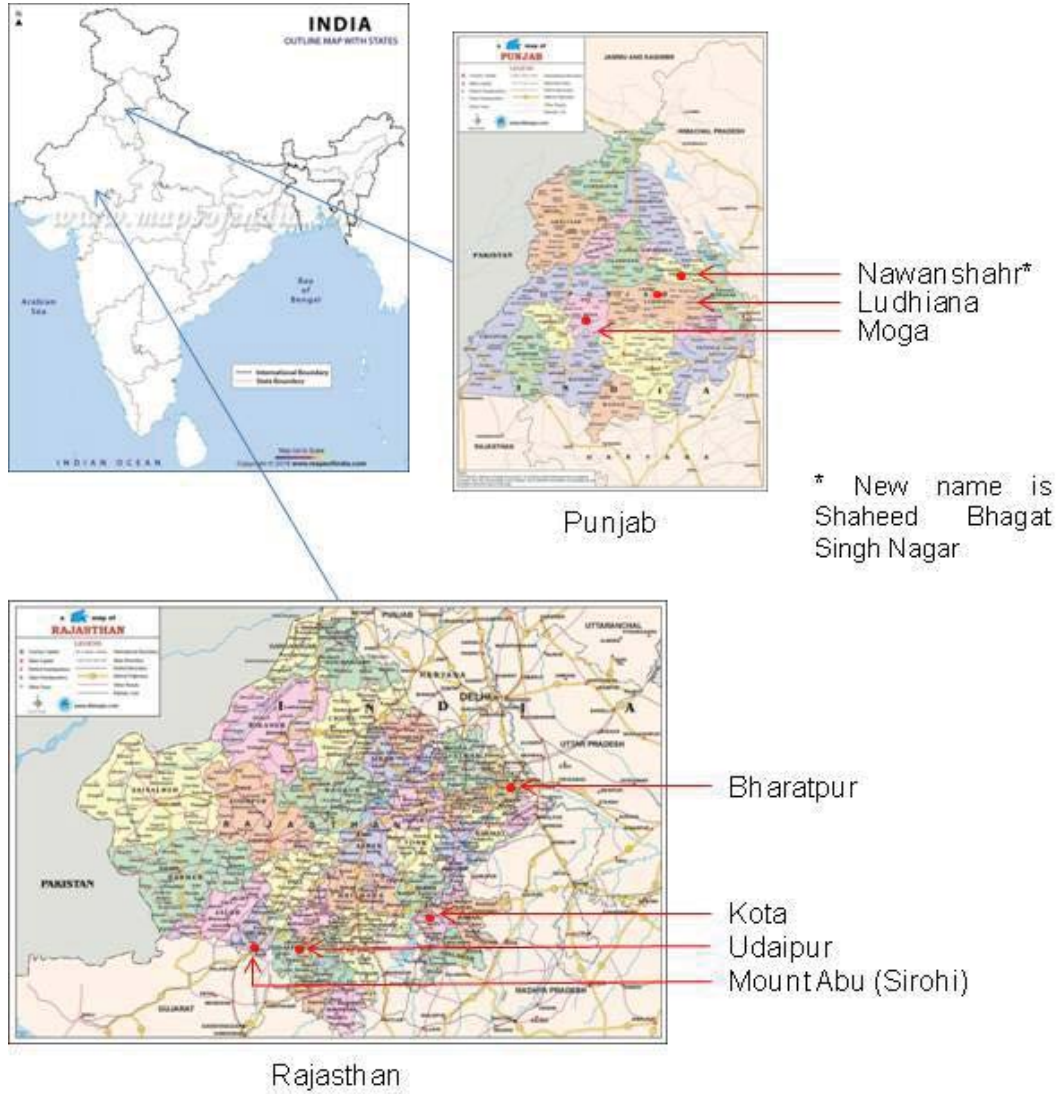

Figure 1: Location map of the investigation sites.

and four aquatic habitats in form of waste water collection ponds or effluent/ sewage treatment plant (A). The habitats as per the sites are given in Table 1.

\section{Methodology}

Over all, eleven sites of seven urban areas were visited by the authors in different seasons over the year (Jan 2016 - Jan 2017).

The surveys and scientific samplings of the investigation area were started from 1999 onwards from Udaipur, 2002 onwards from Mount Abu, 2007 onwards from Bharatpur and 2014 onwards from rest of the sites. The present observations includes records from all the sites for the year 2016 but include past observations also to enlist all the bird species which show their presence on waste disposal sites. Direct sighting records at the site and identification of the species were done during the observations.

The terrestrial habitat sites are symbolized by " $\mathrm{T}$ " and aquatic habitat sites are symbolized by "A" in the present observations. The symbol $\mathrm{T}$ or $\mathrm{A}$ is having serial number for each site of observations (Table 1).

Out of the eleven sites, Mount Abu site (T3) is not in existence due to shifting of the dumping ground from Mount Abu to foothills at Abu Road. Therefore, past records from the Chimney site (T3) of Mount Abu were undertaken by authors. Nomenclature is used as per Manakadan and Pittie [12]. Habitat wise checklist is prepared as per Kumar et al. [13].

\section{Observations and Results}

\section{Species richness and status}

Around 103 bird species belonging to 37 families in 11 orders were recorded from the study area (Tables 2 and 3). Based on the reviews and past observations of the states of Rajasthan and Punjab in general, the status of the species in the investigation area shows that 58 species (ca 56\%) are residents, 18 species are migrants and rest 27 resident species have local migratory nature. Specifically, there are slight differences in the status of the species at local levels due to local movements as per the climatic changes. From the present investigation and recent time period maximum 86 bird species were recorded from sites of Udaipur followed by 75 from Bharatpur sites. Three species in each case was enlisted from the past records. The observations of Chimney Dumping Ground (Mt $\mathrm{Abu}$ ) are based on past records [11].

\section{Species richness and habitat}

The investigated area has diversity of the habitat. Broadly bird species were categorized on the basis of the use of two major habitats, namely terrestrial and aquatic (Table 3). Of the total 103 species, 53 were terrestrial species, 37 wetland species and 13 wetland dependent species.

\section{Globally important species}

As according to the IUCN Red List, three vulture species, namely 
Citation: Mehra SP, Mehra S, Uddin M, Verma V, Sharma H, et al. (2017) Waste as a Resource for Avifauna: Review and Survey of the Avifaunal Composition in and around Waste Dumping Sites and Sewage Water Collection Sites (India). Int J Waste Resour 7: 289. doi: 10.4172/22525211.1000289

Page 3 of 8

\begin{tabular}{|c|c|c|c|c|}
\hline Sr. No. & Urban Area with waste disposal site/s & Site/s of observations & Code Used & Major Type of Habitat \\
\hline & \multicolumn{4}{|c|}{ Rajasthan } \\
\hline 1 & \multirow{2}{*}{ Bharatpur } & Dumping site near $\mathrm{NH} 11$ & T1 & Terrestrial \\
\hline 2 & & Scattered ponds and nallahs (drains) & $\mathrm{A} 1$ & Aquatic \\
\hline 3 & \multirow{2}{*}{ Kota } & Garbage Dumping Ground & $\mathrm{T} 2$ & Terrestrial \\
\hline 4 & & Thermal Ash Pond & A2 & Aquatic \\
\hline 5 & Mt Abu & Chimney Site & T3 & Terrestrial \\
\hline 6 & \multirow{3}{*}{ Udaipur } & Baleecha Dumping Site & $\mathrm{T} 4$ & Terrestrial \\
\hline 7 & & Tetardi Dumping Site & T5 & Terrestrial \\
\hline \multirow[t]{2}{*}{8} & & Ahar nallah (drain) & A3 & Aquatic \\
\hline & \multicolumn{4}{|c|}{ Punjab } \\
\hline 9 & Moga & Garbage Dumping Ground & T6 & Terrestrial \\
\hline 10 & $\begin{array}{c}\text { Nawanshahr (Shaheed Bhagat Singh } \\
\text { Nagar) }\end{array}$ & Garbage Dumping Ground & $\mathrm{T} 7$ & Terrestrial \\
\hline 11 & Ludhiana & Budha nallah (drain) & A4 & Aquatic \\
\hline
\end{tabular}

Table 1: Habitats as per the sites.

\begin{tabular}{|c|c|c|c|c|c|c|c|c|c|c|}
\hline \multirow[b]{2}{*}{$\begin{array}{c}\text { Sr. } \\
\text { No. }\end{array}$} & \multirow[b]{2}{*}{ Common Name } & \multirow[b]{2}{*}{ Scientific Name } & \multirow[b]{2}{*}{$\begin{array}{l}\frac{\pi}{\pi} \\
\frac{1}{0} \\
\frac{0}{1} \\
\frac{1}{1}\end{array}$} & \multicolumn{7}{|c|}{ Observations from the Urban Areas } \\
\hline & & & & $\begin{array}{l}\frac{1}{3} \\
\frac{2}{\pi} \\
\frac{\pi}{\pi} \\
\frac{5}{\infty}\end{array}$ & ث̊ & $\begin{array}{l}{ }^{*} \\
\frac{0}{2} \\
\frac{1}{\Sigma} \\
\Sigma\end{array}$ & $\frac{\grave{2}}{\frac{2}{\pi}}$ & $\begin{array}{l}\text { న్ } \\
\frac{0}{\Sigma}\end{array}$ & $\begin{array}{l}z \\
\text { 心 }\end{array}$ & $\frac{\pi}{\frac{\pi}{\pi}} \frac{\pi}{\frac{\pi}{2}}$ \\
\hline 1 & Grebes & Podicipedidae & & & & & & & & \\
\hline 1 & Little Grebe (5) & $\begin{array}{c}\text { Tachybaptus ruficollis (Pallas, } \\
1764 \text { ) }\end{array}$ & $\mathbf{w}$ & $x$ & $\mathrm{x}$ & $x^{0}$ & $x$ & $\mathrm{x}$ & $\mathrm{x}$ & $\mathrm{x}$ \\
\hline 2 & Cormorants/ Shags & Phalacrocoracidae & & & & & & & & \\
\hline 2 & Little Cormorant (28) & Phalacrocorax niger (Vieillot, 1817) & $\mathbf{w}$ & $x$ & 0 & 0 & $x$ & 0 & 0 & 0 \\
\hline 3 & Herons, Egrets and Bitterns & Ardeidae & & & & & & & & \\
\hline 3 & Little Egret (49) & Egretta garzetta (Linnaeus, 1766) & $\mathbf{w}$ & $x$ & $x$ & $x^{0}$ & $x$ & $x$ & $x$ & $\mathrm{x}$ \\
\hline 4 & $\begin{array}{l}\text { Large Egret or Great Egret } \\
\qquad(45-46)\end{array}$ & $\begin{array}{c}\text { Casmerodius albus (Linnaeus, } \\
1758)\end{array}$ & $\mathbf{w}$ & $\mathrm{x}$ & 0 & $x^{\circ}$ & $x$ & 0 & 0 & $x$ \\
\hline 5 & $\begin{array}{c}\text { Median Egret or Intermediate } \\
\text { Egret }(47,48)\end{array}$ & $\begin{array}{c}\text { Mesophoyx intermedia (Wagler, } \\
\text { 1829) }\end{array}$ & $\mathbf{w}$ & $x$ & $x$ & $x^{\circ}$ & $x$ & 0 & 0 & $x$ \\
\hline 6 & Cattle Egret (44) & Bubulcus ibis (Linnaeus, 1758) & $\mathbf{W}$ & $x$ & $x$ & $x^{0}$ & $x$ & $x$ & $x$ & $\mathrm{x}$ \\
\hline 7 & Indian Pond-Heron (42-42a) & Ardeola grayii (Sykes, 1832) & $\mathbf{w}$ & $x$ & $\mathrm{x}$ & $x^{\circ}$ & $x$ & $x$ & $x$ & $x$ \\
\hline 8 & $\begin{array}{l}\text { Little Green Heron or Little } \\
\text { Heron }(38-41)\end{array}$ & Butorides striatus (Linnaeus, 1758) & $\mathbf{W}$ & $x$ & 0 & 0 & $x$ & 0 & 0 & 0 \\
\hline 9 & $\begin{array}{l}\text { Black-crowned Night-Heron } \\
(52)\end{array}$ & $\begin{array}{c}\text { Nycticorax nycticorax (Linnaeus, } \\
1758)\end{array}$ & $\mathbf{W}$ & $x$ & 0 & 0 & $x$ & 0 & 0 & 0 \\
\hline 4 & Storks & Ciconiidae & & & & & & & & \\
\hline 10 & Painted Stork (60) & $\begin{array}{c}\text { Mycteria leucocephala (Pennant, } \\
\text { 1769) }\end{array}$ & $\mathbf{w}$ & $x$ & 0 & 0 & $x$ & 0 & 0 & 0 \\
\hline 11 & $\begin{array}{c}\text { Asian Openbill-Stork or Asian } \\
\text { Openbill (61) }\end{array}$ & $\begin{array}{c}\text { Anastomus oscitans (Boddaert, } \\
1783 \text { ) }\end{array}$ & $\mathbf{w}$ & $x$ & 0 & 0 & $x$ & 0 & 0 & 0 \\
\hline 5 & Ibises and Spoonbills & Threskiornithidae & & & & & & & & \\
\hline 12 & Glossy Ibis (71) & $\begin{array}{l}\text { Plegadis falcinellus (Linnaeus, } \\
1766 \text { ) }\end{array}$ & $\mathbf{w}$ & $x$ & $x$ & $x^{\circ}$ & $x$ & 0 & 0 & 0 \\
\hline 13 & $\begin{array}{c}\text { Oriental White Ibis or Black- } \\
\text { headed Ibis }(69)\end{array}$ & $\begin{array}{l}\text { Threskiornis melanocephalus } \\
\text { (Latham, 1790) }\end{array}$ & $\mathbf{w}$ & $x$ & $x$ & 0 & $x$ & 0 & 0 & 0 \\
\hline 14 & Black Ibis $(70)$ & $\begin{array}{c}\text { Pseudibis papillosa (Temminck, } \\
\text { 1824) }\end{array}$ & $\mathbf{w}$ & $x$ & $x$ & $x^{0}$ & $x$ & 0 & 0 & 0 \\
\hline 6 & Geese and Ducks & Anatidae & & & & & & & & \\
\hline 15 & Lesser Whistling-Duck (88) & $\begin{array}{c}\text { Dendrocygna javanica (Horsfield, } \\
\text { 1821) }\end{array}$ & $\mathbf{w}$ & $x$ & $x$ & 0 & $x$ & 0 & 0 & 0 \\
\hline 16 & $\begin{array}{c}\text { Brahminy Shelduck or Ruddy } \\
\text { Shelduck (90) }\end{array}$ & Tadorna ferruginea (Pallas, 1764) & $\mathbf{w}$ & $x$ & 0 & 0 & $x$ & 0 & 0 & 0 \\
\hline 17 & Comb Duck (115) & $\begin{array}{l}\text { Sarkidiornis melanotos (Pennant, } \\
1769 \text { ) }\end{array}$ & W & $x$ & 0 & 0 & $x$ & 0 & 0 & 0 \\
\hline 18 & Northern Shoveller (105) & Anas clypeata Linnaeus, 1758 & $\mathbf{w}$ & $x$ & 0 & 0 & $x$ & 0 & 0 & 0 \\
\hline 7 & $\begin{array}{c}\text { Hawks, Eagles, Buzzards, } \\
\text { Old World Vultures, Kites, } \\
\text { Harriers }\end{array}$ & Accipitridae & & & & & & & & \\
\hline
\end{tabular}


Citation: Mehra SP, Mehra S, Uddin M, Verma V, Sharma H, et al. (2017) Waste as a Resource for Avifauna: Review and Survey of the Avifaunal Composition in and around Waste Dumping Sites and Sewage Water Collection Sites (India). Int J Waste Resour 7: 289. doi: 10.4172/22525211.1000289

Page 4 of 8

\begin{tabular}{|c|c|c|c|c|c|c|c|c|c|c|}
\hline 19 & Black-shouldered Kite (124 & $\begin{array}{c}\text { Elanus caeruleus (Desfontaines, } \\
1789)\end{array}$ & $\mathbf{T}$ & $x$ & $x$ & $x^{\circ}$ & $x$ & $x$ & $x$ & $\mathrm{x}$ \\
\hline 20 & Black Kite (132-134) & Milvus migrans (Boddaert, 1783) & $\mathbf{T}$ & $\mathrm{x}$ & $\mathrm{x}$ & $x^{\circ}$ & $\mathrm{x}$ & $\mathrm{x}$ & $x$ & $x$ \\
\hline 21 & Brahminy Kite (135) & Haliastur indus (Boddaert, 1783) & WD & $x$ & 0 & 0 & $\mathrm{x}$ & 0 & 0 & 0 \\
\hline 22 & Egyptian Vulture (186-187) & $\begin{array}{c}\text { Neophron percnopterus (Linnaeus, } \\
1758 \text { ) }\end{array}$ & $\mathbf{T}$ & $x$ & 0 & $x^{\circ}$ & $x$ & 0 & 0 & 0 \\
\hline 23 & $\begin{array}{l}\text { Indian White-backed Vulture or } \\
\text { White-rumped Vulture (185) }\end{array}$ & Gyps bengalensis (Gmelin, 1788) & $\mathbf{T}$ & $x^{\circ}$ & 0 & 0 & $x^{\circ}$ & 0 & 0 & 0 \\
\hline 24 & Long-billed Vulture (182) & Gyps indicus (Scopoli, 1786) & $\mathbf{T}$ & $x^{\circ}$ & 0 & 0 & $x^{\circ}$ & 0 & 0 & 0 \\
\hline 25 & Red-headed Vulture (178) & Sarcogyps calvus (Scopoli, 1786) & $\mathbf{T}$ & $x^{0}$ & 0 & 0 & $x^{\circ}$ & 0 & 0 & 0 \\
\hline 26 & $\begin{array}{l}\text { Western Marsh-Harrier or } \\
\text { Eurasian Marsh Harrier (193) }\end{array}$ & $\begin{array}{l}\text { Circus aeruginosus (Linnaeus, } \\
1758)\end{array}$ & WD & $\mathrm{x}$ & 0 & 0 & $\mathrm{x}$ & 0 & 0 & 0 \\
\hline 27 & Shikra $(137-140)$ & Accipiter badius (Gmelin, 1788) & $\mathbf{T}$ & $x$ & $x$ & $x^{\circ}$ & $x$ & $x$ & $x$ & $x$ \\
\hline 28 & Steppe Eagle (169) & Aquila nipalensis Hodgson, 1833 & WD & 0 & 0 & 0 & $x$ & 0 & 0 & 0 \\
\hline 29 & Eastern Imperial Eagle (167) & Aquila heliaca Savigny, 1809 & WD & 0 & 0 & 0 & $\mathrm{x}$ & 0 & 0 & 0 \\
\hline 8 & Osprey & Pandionidae & & & & & & & & \\
\hline 30 & Osprey (203) & Pandion haliaetus (Linnaeus, 1758) & WD & 0 & 0 & 0 & $\mathrm{x}$ & 0 & 0 & 0 \\
\hline 9 & Falcons & Falconidae & & & & & & & & \\
\hline 31 & Lesser Kestrel (221) & Falco naumanni Fleischer, 1818 & $\mathbf{T}$ & 0 & 0 & $x^{\circ}$ & 0 & 0 & 0 & 0 \\
\hline 32 & Common Kestrel (222-224) & Falco tinnunculus Linnaeus, 1758 & $\mathbf{T}$ & 0 & 0 & $x^{\circ}$ & $x$ & 0 & 0 & 0 \\
\hline 33 & $\begin{array}{l}\text { Red-headed Falcon or Red- } \\
\text { necked Falcon (219) }\end{array}$ & Falco chicquera Daudin, 1800 & $\mathbf{T}$ & 0 & 0 & $x^{\circ}$ & 0 & 0 & 0 & 0 \\
\hline 34 & Laggar or Laggar Falcon (208) & Falco jugger J.E. Gray, 1834 & $\mathbf{T}$ & 0 & 0 & $x^{\circ}$ & 0 & 0 & 0 & 0 \\
\hline 35 & Peregrine Falcon (209-211) & Falco peregrinus Tunstall, 1771 & WD & $\mathrm{x}$ & 0 & 0 & 0 & 0 & 0 & 0 \\
\hline 10 & $\begin{array}{c}\text { Pheasants, Partridges, } \\
\text { Quails }\end{array}$ & Phasianidae & & & & & & & & \\
\hline 36 & Grey Francolin (244-246) & $\begin{array}{c}\text { Francolinus pondicerianus (Gmelin, } \\
\text { 1789) }\end{array}$ & $\mathbf{T}$ & $\mathrm{x}$ & 0 & $x^{\circ}$ & $x$ & 0 & 0 & 0 \\
\hline 37 & Rain Quail (252) & $\begin{array}{c}\text { Coturnix coromandelica (Gmelin, } \\
1789)\end{array}$ & $\mathbf{T}$ & 0 & 0 & $x^{\circ}$ & 0 & 0 & 0 & 0 \\
\hline 38 & Jungle Bush-Quail (255-258) & Perdicula asiatica (Latham, 1790) & $\mathbf{T}$ & 0 & 0 & $x^{\circ}$ & $\mathrm{x}$ & 0 & 0 & 0 \\
\hline 39 & Rock Bush-Quail (259-261) & Perdicula argoondah (Sykes, 1832) & $\mathbf{T}$ & 0 & 0 & $x^{\circ}$ & $x$ & 0 & 0 & 0 \\
\hline 40 & Grey Junglefowl (301) & Gallus sonneratii Temminck, 1813 & $\mathbf{T}$ & 0 & 0 & $x^{\circ}$ & 0 & 0 & 0 & 0 \\
\hline 41 & Indian Peafowl (311) & Pavo cristatus Linnaeus, 1758 & $\mathbf{T}$ & $x$ & $\mathrm{x}$ & $x^{\circ}$ & $x$ & $x$ & $x$ & $x$ \\
\hline 11 & $\begin{array}{l}\text { Rails, Crakes, Moorhens, } \\
\text { Coots }\end{array}$ & Rallidae & & & & & & & & \\
\hline 42 & $\begin{array}{l}\text { White-breasted Waterhen } \\
(343-345)\end{array}$ & $\begin{array}{c}\text { Amaurornis phoenicurus (Pennant, } \\
1769 \text { ) }\end{array}$ & $\mathbf{w}$ & $\mathrm{x}$ & $\mathrm{x}$ & $x^{\circ}$ & $x$ & 0 & 0 & $x$ \\
\hline 43 & $\begin{array}{l}\text { Purple Moorhen or Purple } \\
\text { Swamphen (348-349) }\end{array}$ & $\begin{array}{c}\text { Porphyrio porphyrio (Linnaeus, } \\
1758 \text { ) }\end{array}$ & $\mathbf{w}$ & $x$ & $x$ & $x^{\circ}$ & $x$ & 0 & 0 & $\mathrm{x}$ \\
\hline 44 & Common Moorhen (347-347a) & $\begin{array}{l}\text { Gallinula chloropus (Linnaeus, } \\
1758 \text { ) }\end{array}$ & $\mathbf{w}$ & $x$ & $\mathrm{x}$ & $x^{\circ}$ & $\mathrm{x}$ & 0 & 0 & $x$ \\
\hline 45 & Common Coot (350) & Fulica atra Linnaeus, 1758 & $\mathbf{w}$ & $\mathrm{x}$ & $\mathrm{x}$ & $x^{\circ}$ & $\mathrm{x}$ & 0 & 0 & $x$ \\
\hline 12 & Jacanas & Jacanidae & & & & & & & & \\
\hline 46 & Pheasant-tailed Jacana (358) & $\begin{array}{c}\text { Hydrophasianus chirurgus (Scopoli, } \\
\text { 1786) }\end{array}$ & $\mathbf{w}$ & $\mathrm{x}$ & $x$ & $x^{\circ}$ & $x$ & 0 & 0 & 0 \\
\hline 47 & Bronze-winged Jacana (359) & Metopidius indicus (Latham, 1790) & $\mathbf{w}$ & $x$ & $x$ & $x^{\circ}$ & $\mathrm{x}$ & 0 & 0 & 0 \\
\hline 13 & Painted-Snipes & Rostratulidae & & & & & & & & \\
\hline 48 & Greater Painted-Snipe (429) & $\begin{array}{c}\text { Rostratula benghalensis (Linnaeus, } \\
1758 \text { ) }\end{array}$ & $\mathbf{w}$ & $x$ & 0 & $x^{\circ}$ & $x$ & 0 & 0 & 0 \\
\hline 14 & Plovers, Lapwings & Charadriidae & & & & & & & & \\
\hline 49 & Little Ringed Plover (379-380) & Charadrius dubius Scopoli, 1786 & $\mathbf{w}$ & $\mathrm{x}$ & $x$ & $x^{\circ}$ & $x$ & 0 & 0 & 0 \\
\hline 50 & $\begin{array}{l}\text { Red-wattled Lapwing (366- } \\
\text { 368) }\end{array}$ & Vanellus indicus (Boddaert, 1783) & $\mathbf{w}$ & $x$ & $x$ & $x^{\circ}$ & $\mathrm{x}$ & $x$ & $x$ & $\mathrm{x}$ \\
\hline 15 & $\begin{array}{l}\text { Sandpipers, Stints, Snipes, } \\
\text { Godwits and Curlews }\end{array}$ & Scolopacidae & & & & & & & & \\
\hline 51 & Common Snipe (409) & $\begin{array}{c}\text { Gallinago gallinago (Linnaeus, } \\
1758 \text { ) }\end{array}$ & $\mathbf{w}$ & 0 & 0 & 0 & $x$ & 0 & 0 & 0 \\
\hline 52 & Black-tailed Godwit (389-390) & Limosa limosa (Linnaeus, 1758) & $\mathbf{w}$ & $\mathrm{x}$ & $\mathrm{x}$ & 0 & $\mathrm{x}$ & 0 & 0 & 0 \\
\hline 53 & Common Redshank $(393,394)$ & Tringa totanus (Linnaeus, 1758) & $\mathbf{w}$ & $\mathrm{x}$ & $x$ & 0 & $\mathrm{x}$ & 0 & 0 & 0 \\
\hline 54 & Wood Sandpiper (398) & Tringa glareola Linnaeus, 1758 & $\mathbf{W}$ & $x$ & 0 & 0 & $x$ & 0 & 0 & 0 \\
\hline 55 & Common Sandpiper (401) & Actitis hypoleucos Linnaeus, 1758 & $\mathbf{w}$ & $x$ & 0 & 0 & $x$ & 0 & 0 & 0 \\
\hline 56 & Little Stint (416) & Calidris minuta (Leisler, 1812) & $\mathbf{w}$ & $x$ & 0 & 0 & $x$ & 0 & 0 & 0 \\
\hline
\end{tabular}


Citation: Mehra SP, Mehra S, Uddin M, Verma V, Sharma H, et al. (2017) Waste as a Resource for Avifauna: Review and Survey of the Avifaunal Composition in and around Waste Dumping Sites and Sewage Water Collection Sites (India). Int J Waste Resour 7: 289. doi: 10.4172/22525211.1000289

Page 5 of 8

\begin{tabular}{|c|c|c|c|c|c|c|c|c|c|c|}
\hline 57 & Ruff (426) & $\begin{array}{l}\text { Philomachus pugnax (Linnaeus, } \\
\text { 1758) }\end{array}$ & $\mathbf{w}$ & $x$ & 0 & 0 & $x$ & 0 & 0 & 0 \\
\hline 16 & Avocets and Stilts & Recurvirostridae & & & & & & & & \\
\hline 58 & Black-winged Stilt (430-431) & $\begin{array}{l}\text { Himantopus himantopus (Linnaeus, } \\
\text { 1758) }\end{array}$ & $\mathbf{w}$ & $x$ & 0 & 0 & $x$ & 0 & 0 & $\mathrm{x}$ \\
\hline 17 & $\begin{array}{l}\text { Stone-Curlew and Stone- } \\
\text { Plovers/Thick-knees }\end{array}$ & Burhinidae & & & & & & & & \\
\hline 59 & $\begin{array}{l}\text { Stone-Curlew or Eurasian } \\
\text { Thick-knee (435-436) }\end{array}$ & $\begin{array}{l}\text { Burhinus oedicnemus (Linnaeus, } \\
\text { 1758) }\end{array}$ & $\mathbf{T}$ & $x$ & 0 & 0 & $x$ & 0 & 0 & 0 \\
\hline 60 & $\begin{array}{l}\text { Great Stone-Plover or Great } \\
\text { Thick-knee (437) }\end{array}$ & Esacus recurvirostris (Cuvier, 1829) & $\mathbf{w}$ & 0 & 0 & 0 & $x$ & 0 & 0 & 0 \\
\hline 18 & Gulls, Terns & Laridae & & & & & & & & \\
\hline 61 & River Tern (463) & Sterna aurantia J.E. Gray, 1831 & $\mathbf{w}$ & $\mathrm{x}$ & 0 & 0 & $x$ & 0 & 0 & 0 \\
\hline 19 & Pigeons and Doves & Columbidae & & & & & & & & \\
\hline 62 & Blue Rock Pigeon (516-517) & Columba livia Gmelin, 1789 & $\mathbf{T}$ & $\mathrm{x}$ & $x$ & $x^{\circ}$ & $x$ & $x$ & $\mathrm{x}$ & 0 \\
\hline 63 & $\begin{array}{c}\text { Little Brown Dove or Laughing } \\
\text { Dove (541) }\end{array}$ & $\begin{array}{l}\text { Streptopelia senegalensis } \\
\quad(\text { Linnaeus, 1766) }\end{array}$ & $\mathbf{T}$ & $\mathrm{x}$ & $x$ & $x^{\circ}$ & $x$ & $\mathrm{x}$ & $x$ & 0 \\
\hline 64 & Red Collared-Dove (535-536) & $\begin{array}{l}\text { Streptopelia tranquebarica } \\
\text { (Hermann, 1804) }\end{array}$ & $\mathbf{T}$ & $x$ & $x$ & $x^{\circ}$ & $\mathrm{x}$ & $\mathrm{x}$ & $\mathrm{x}$ & 0 \\
\hline 65 & Eurasian Collared-Dove (534) & $\begin{array}{c}\text { Streptopelia decaocto (Frivaldszky, } \\
\text { 1838) }\end{array}$ & $\mathbf{T}$ & $x$ & $x$ & $x^{0}$ & $x$ & $\mathrm{x}$ & $x$ & 0 \\
\hline 20 & Parakeets & Psittacidae & & & & & & & & \\
\hline 66 & $\begin{array}{l}\text { Rose-ringed Parakeet (549- } \\
550)\end{array}$ & Psittacula krameri (Scopoli, 1769) & $\mathbf{T}$ & $x$ & $x$ & $x^{0}$ & $x$ & 0 & 0 & 0 \\
\hline 21 & $\begin{array}{l}\text { Cuckoos, Malkohas and } \\
\text { Coucals }\end{array}$ & Cuculidae & & & & & & & & \\
\hline 67 & Greater Coucal (600-602) & $\begin{array}{c}\text { Centropus sinensis (Stephens, } \\
\text { 1815) }\end{array}$ & $\mathbf{T}$ & $x$ & $x$ & $x^{\circ}$ & $x$ & 0 & 0 & 0 \\
\hline 22 & Owls & Strigidae & & & & & & & & \\
\hline 68 & Spotted Owlet (650-652) & Athene brama (Temminck, 1821) & $\mathbf{T}$ & 0 & 0 & $x^{\circ}$ & $x$ & 0 & 0 & 0 \\
\hline 23 & Kingfishers & Alcedinidae & & & & & & & & \\
\hline 69 & $\begin{array}{c}\text { Small Blue Kingfisher or } \\
\text { Common Kingfisher (722-724) }\end{array}$ & Alcedo atthis (Linnaeus, 1758) & WD & $x$ & 0 & 0 & $x$ & 0 & 0 & 0 \\
\hline 70 & $\begin{array}{c}\text { White-breasted Kingfisher } \\
\text { or White-throated Kingfisher } \\
(735-738)\end{array}$ & $\begin{array}{l}\text { Halcyon smyrnensis (Linnaeus, } \\
1758)\end{array}$ & WD & $\mathrm{x}$ & $x$ & $x^{\circ}$ & $x$ & $x$ & $x$ & $\mathrm{x}$ \\
\hline 24 & Bee-eaters & Meropidae & & & & & & & & \\
\hline 71 & Small Bee-eater (749-752) & Merops orientalis Latham, 1801 & $\mathbf{T}$ & $x$ & $x$ & $x^{0}$ & $x$ & $x$ & $x$ & 0 \\
\hline 25 & Rollers & Coraciidae & & & & & & & & \\
\hline 72 & Indian Roller (755-757) & $\begin{array}{c}\text { Coracias benghalensis (Linnaeus, } \\
1758 \text { ) }\end{array}$ & $\mathbf{T}$ & $x$ & 0 & $x^{\circ}$ & $x$ & 0 & 0 & 0 \\
\hline 26 & Hoopoes & Upupidae & & & & & & & & \\
\hline 73 & Common Hoopoe (763-766) & Upupa epops Linnaeus, 1758 & $\mathbf{T}$ & $\mathrm{x}$ & $\mathrm{x}$ & $x^{0}$ & $x$ & 0 & 0 & 0 \\
\hline 27 & Swallows and Martins & Hirundinidae & & & & & & & & \\
\hline 74 & Dusky Crag-Martin (914) & Hirundo concolor Sykes, 1833 & $\mathbf{T}$ & $\mathrm{x}$ & 0 & $x^{\circ}$ & $x$ & 0 & 0 & 0 \\
\hline 75 & Wire-tailed Swallow (921) & Hirundo smithii Leach, 1818 & WD & $\mathrm{x}$ & $x$ & $x^{\circ}$ & $x$ & 0 & 0 & 0 \\
\hline 76 & $\begin{array}{l}\text { Red-rumped Swallow (923- } \\
\text { 928) }\end{array}$ & Hirundo daurica Linnaeus, 1771 & WD & $\mathrm{x}$ & 0 & $x^{0}$ & $x$ & 0 & 0 & 0 \\
\hline 28 & Wagtails and Pipits & Motacillidae & & & & & & & & \\
\hline 77 & White Wagtail (1885-1890) & Motacilla alba Linnaeus, 1758 & WD & $\mathrm{x}$ & 0 & $x^{\circ}$ & $x$ & 0 & 0 & 0 \\
\hline 78 & $\begin{array}{l}\text { Large Pied Wagtail or White- } \\
\text { browed Wagtail (1891) }\end{array}$ & $\begin{array}{c}\text { Motacilla maderaspatensis Gmelin, } \\
1789\end{array}$ & WD & $x$ & $x$ & $x^{0}$ & $x$ & 0 & 0 & 0 \\
\hline 79 & Grey Wagtail (1884) & Motacilla cinerea Tunstall, 1771 & WD & $\mathrm{x}$ & 0 & $x^{\circ}$ & $x$ & 0 & 0 & 0 \\
\hline 29 & Bulbuls & Pycnonotidae & & & & & & & & \\
\hline 80 & $\begin{array}{l}\text { White-eared Bulbul (1123- } \\
\text { 1124) }\end{array}$ & Pycnonotus leucotis (Gould, 1836) & $\mathbf{T}$ & 0 & $\mathrm{x}$ & 0 & 0 & 0 & 0 & 0 \\
\hline 81 & Red-vented Bulbul (1126-1132) & Pycnonotus cafer (Linnaeus, 1766) & $\mathbf{T}$ & $\mathrm{x}$ & $\mathrm{x}$ & $x^{\circ}$ & $\mathrm{x}$ & $x$ & $x$ & 0 \\
\hline 30 & Shrikes & Laniidae & & & & & & & & \\
\hline 82 & Rufous-tailed Shrike (942-943) & $\begin{array}{c}\text { Lanius isabellinus Hemprich and } \\
\text { Ehrenberg, } 1833\end{array}$ & $\mathbf{T}$ & 0 & 0 & $x^{\circ}$ & 0 & 0 & 0 & 0 \\
\hline 83 & Brown Shrike (949-950a) & Lanius cristatus Linnaeus, 1758 & $\mathbf{T}$ & 0 & 0 & $x^{\circ}$ & 0 & 0 & 0 & 0 \\
\hline 84 & Bay-backed Shrike (939-940) & Lanius vittatus Valenciennes, 1826 & $\mathbf{T}$ & 0 & 0 & $x^{\circ}$ & $\mathrm{x}$ & 0 & 0 & 0 \\
\hline 85 & $\begin{array}{c}\text { Rufous-backed Shrike or Long- } \\
\text { tailed Shrike }(946-948)\end{array}$ & Lanius schach Linnaeus, 1758 & $\mathbf{T}$ & 0 & 0 & $x^{\circ}$ & 0 & 0 & 0 & 0 \\
\hline
\end{tabular}


Citation: Mehra SP, Mehra S, Uddin M, Verma V, Sharma H, et al. (2017) Waste as a Resource for Avifauna: Review and Survey of the Avifaunal Composition in and around Waste Dumping Sites and Sewage Water Collection Sites (India). Int J Waste Resour 7: 289. doi: 10.4172/22525211.1000289

Page 6 of 8

\begin{tabular}{|c|c|c|c|c|c|c|c|c|c|c|}
\hline 86 & $\begin{array}{l}\text { Southern Grey Shrike (933- } \\
\text { 935) }\end{array}$ & $\begin{array}{l}\text { Lanius meridionalis Temminck, } \\
1820\end{array}$ & $\mathbf{T}$ & 0 & 0 & $x^{0}$ & $x$ & 0 & 0 & 0 \\
\hline 31 & Thrushes, Robins, Wheaters & Turdinae & & & & & & & & \\
\hline 87 & $\begin{array}{l}\text { Oriental Magpie-Robin (1661- } \\
1664)\end{array}$ & $\begin{array}{c}\text { Copsychus saularis (Linnaeus, } \\
1758 \text { ) }\end{array}$ & $\mathbf{T}$ & $x$ & $x$ & $x^{\circ}$ & $x$ & 0 & 0 & 0 \\
\hline 88 & Indian Robin (1717-1721) & $\begin{array}{l}\text { Saxicoloides fulicata (Linnaeus, } \\
\text { 1776) }\end{array}$ & $\mathbf{T}$ & $x$ & $x$ & $x^{\circ}$ & $x$ & $x$ & $x$ & 0 \\
\hline 89 & Black Redstart (1671-1672) & $\begin{array}{c}\text { Phoenicurus ochruros (Gmelin, } \\
\text { 1774) }\end{array}$ & $\mathbf{T}$ & $x$ & 0 & $x^{\circ}$ & $x$ & 0 & 0 & 0 \\
\hline 90 & Indian Chat (1692) & Cercomela fusca (Blyth, 1851) & $\mathbf{T}$ & $x$ & $x$ & $x^{0}$ & $x$ & $x$ & $x$ & 0 \\
\hline 32 & Babblers & Timaliinae & & & & & & & & \\
\hline 91 & $\begin{array}{c}\text { Rufous-bellied Babbler or } \\
\text { Tawny-bellied Babbler (1219- } \\
\text { 1223) }\end{array}$ & $\begin{array}{c}\text { Dumetia hyperythra (Franklin, } \\
1831)\end{array}$ & $\mathbf{T}$ & 0 & 0 & $x^{\circ}$ & 0 & 0 & 0 & 0 \\
\hline 92 & Large Grey Babbler (1258) & Turdoides malcolmi (Sykes, 1832) & $\mathbf{T}$ & $x$ & 0 & $x^{\circ}$ & $x$ & 0 & 0 & 0 \\
\hline 93 & Jungle Babbler (1261-1265) & Turdoides striatus (Dumont, 1823) & $\mathbf{T}$ & $x$ & 0 & $x^{\circ}$ & $x$ & 0 & 0 & 0 \\
\hline 33 & Munias (Estrildid Finches) & Estrildidae & & & & & & & & \\
\hline 94 & $\begin{array}{c}\text { White-throated Munia or Indian } \\
\text { Silverbill (1966) }\end{array}$ & $\begin{array}{l}\text { Lonchura malabarica (Linnaeus, } \\
\text { 1758) }\end{array}$ & $\mathbf{T}$ & 0 & 0 & $x^{\circ}$ & 0 & 0 & 0 & 0 \\
\hline 34 & Sparrows & Passerinae & & & & & & & & \\
\hline 95 & House Sparrow (1938-1939a) & $\begin{array}{c}\text { Passer domesticus (Linnaeus, } \\
1758 \text { ) }\end{array}$ & $\mathbf{T}$ & $\mathrm{x}$ & $\mathrm{x}$ & $x^{\circ}$ & $x$ & 0 & $\mathrm{x}$ & 0 \\
\hline 96 & $\begin{array}{c}\text { Yellow-throated Sparrow or } \\
\text { Chestnut-shouldered Petronia } \\
(1948-1949)\end{array}$ & $\begin{array}{c}\text { Petronia xanthocollis (Burton, } \\
\text { 1838) }\end{array}$ & $\mathbf{T}$ & 0 & 0 & $x^{\circ}$ & 0 & 0 & 0 & 0 \\
\hline 35 & Starlings and Mynas & Sturnidae & & & & & & & & \\
\hline 97 & Brahminy Starling (994) & Sturnus pagodarum (Gmelin, 1789) & $\mathbf{T}$ & 0 & 0 & 0 & $x$ & 0 & 0 & 0 \\
\hline 98 & $\begin{array}{c}\text { Asian Pied Starling (1002- } \\
1004)\end{array}$ & Sturnus contra Linnaeus, 1758 & $\mathbf{T}$ & $x$ & $\mathrm{x}$ & $x^{\circ}$ & $x$ & $x$ & $x$ & 0 \\
\hline 99 & Common Myna (1006-1007) & $\begin{array}{c}\text { Acridotheres tristis (Linnaeus, } \\
1766 \text { ) }\end{array}$ & $\mathbf{T}$ & $x$ & $x$ & $x^{\circ}$ & $x$ & $x$ & $\mathrm{x}$ & 0 \\
\hline 100 & Bank Myna (1008) & $\begin{array}{l}\text { Acridotheres ginginianus (Latham, } \\
1790 \text { ) }\end{array}$ & $\mathbf{T}$ & $x$ & $x$ & $x^{\circ}$ & $x$ & $\mathrm{x}$ & $x$ & 0 \\
\hline 36 & Drongos & Dicruridae & & & & & & & & \\
\hline 101 & Black Drongo (962-964) & Dicrurus macrocercus Vieillot, 1817 & $\mathbf{T}$ & $x$ & $x$ & $x^{\circ}$ & $x$ & 0 & 0 & 0 \\
\hline 37 & Crows, Treepies & Corvidae & & & & & & & & \\
\hline 102 & House Crow (1048-1051) & Corvus splendens Vieillot, 1817 & $\mathbf{T}$ & $x$ & $x$ & $x^{\circ}$ & $x$ & $x$ & $x$ & 0 \\
\hline \multirow[t]{2}{*}{103} & $\begin{array}{l}\text { Jungle Crow or Large-billed } \\
\text { Crow (1054-1057) }\end{array}$ & $\begin{array}{l}\text { Corvus macrorhynchos Wagler, } \\
1827\end{array}$ & $\mathbf{T}$ & 0 & 0 & $x^{\circ}$ & 0 & 0 & 0 & 0 \\
\hline & TOTAL & & & 78 & 45 & 70 & 89 & 22 & 23 & 17 \\
\hline \multicolumn{11}{|c|}{$\begin{array}{r}\begin{array}{r}\text { Note: } x=\text { present reco } \\
\text { Mount } A b u^{*}=\text { obser }\end{array} \\
\text { (225-256): Numbers within brackets after the common names are the numbe } \\
T=\text { Terrestrial, } W=V \\
\text { R: Resident; } R M: \text { Resid }\end{array}$} \\
\hline
\end{tabular}

R: Resident; RM: Resident with Local Movement; M: Migrant

Table 2: Checklist of species as per the urban areas.

Indian White-backed Vulture (Gyps bengalensis), Long-billed Vulture (G. indicus) and Red-headed Vulture (Sarcogyps calvus) are the Critically Endangered species, so as the records from the investigation sites. None of the three were sited during the recent year of observations but had the past records from two areas of Udaipur and Bharatpur (Figure 1).

As per the second category of threat i.e., Endangered, two species viz. Egyptian Vulture (Neophron percnopterus) and Steppe Eagle (Aquila nipalensis) were recorded from sites of dumping sites of Bharatpur (Near NH 11 adjoining Keoladeo National Park)) and Udaipur (Baleecha). Eastern Imperial Eagle (Aquila heliaca) enlisted in vulnerable category of threatened species were recorded from site of Udaipur (Baleecha). Seven NT (Near Threatened) species, Painted Stork (Mycteria leucocephala), Black-headed Ibis (Threskiornis melanocephalus), Great Thick Knee (Esacus recurvirostris), Black-tailed Godwit (Limosa lapponica), River Tern (Sterna aurantia), Red-headed Falcon (Falco chicquera), Laggar Falcon (F. jugger) were enlisted in the
Table 2. Out of these five species were recorded from different sites in the investigation period whereas two (Falco sp.) were past records. Thus, dumping (solid and liquid wastes) sites of Udaipur (Baleecha and Ahar Nallah) harbored eight species of global interest whereas sites of Bharatpur were visited by four near threatened species.

\section{Activities and behavior}

The dumping ground was he site with a great variety of food giving space to several micro and macro fauna and flora. These resulted into the food for the bird species. The most common activity observed for the birds was feeding. In raptors, the common feature was single presence except when the animal carcass was part of disposal wastes. The egrets and passerine species were mostly observed in groups. Same was the case with the aquatic species, which were observed in small flocks with different numbers depending on the area of the sewage or effluent waste water collection sites. The stretches in form of nallah 
Citation: Mehra SP, Mehra S, Uddin M, Verma V, Sharma H, et al. (2017) Waste as a Resource for Avifauna: Review and Survey of the Avifaunal Composition in and around Waste Dumping Sites and Sewage Water Collection Sites (India). Int J Waste Resour 7: 289. doi: 10.4172/22525211.1000289

Page 7 of 8

\begin{tabular}{|c|c|c|c|c|c|c|}
\hline \multirow[b]{2}{*}{ Sr. No. } & \multirow[b]{2}{*}{ Orders } & \multirow[b]{2}{*}{ Family } & \multicolumn{4}{|c|}{ Number of Species } \\
\hline & & & Terrestrial & Wetland & Wetland Dependent & $\begin{array}{l}\text { No. of Species in } \\
\text { Study Area }\end{array}$ \\
\hline 1 & Galliformes & Phasianidae & 6 & 0 & 0 & 6 \\
\hline 2 & Anseriformes & Anatidae & 0 & 4 & 0 & 4 \\
\hline 3 & Upupiformes & Upupidae & 1 & 0 & 0 & 1 \\
\hline 4 & Coraciiformes & $\begin{array}{l}\text { Alcedinidae } \\
\text { Meropidae } \\
\text { Coraciidae }\end{array}$ & 2 & 0 & 2 & 4 \\
\hline 5 & Cuculiformes & Cuculidae & 1 & 0 & 0 & 1 \\
\hline 6 & Strigiformes & Strigidae & 1 & 0 & 0 & 1 \\
\hline 7 & Columbiformes & Columbidae & 4 & 0 & 0 & 4 \\
\hline 8 & Psittaciformes & Psittacidae & 1 & 0 & 0 & 1 \\
\hline 9 & Gruiformes & Raliidae & 0 & 4 & 0 & 4 \\
\hline 10 & Ciconiiformes & $\begin{array}{l}\text { Podicipedidae } \\
\text { Phalacrocoracidae } \\
\text { Ardeidae } \\
\text { Ciconiidae } \\
\text { Threskiornithidae } \\
\text { Accipitridae } \\
\text { Pandionidae } \\
\text { Falconidae } \\
\text { Jacanidae } \\
\text { Rostratulidae } \\
\text { Charadriidae } \\
\text { Scolopacidae } \\
\text { Recurvirostridae } \\
\text { Burhinidae } \\
\text { Laridae }\end{array}$ & 12 & 29 & 6 & 47 \\
\hline 11 & Passeriformes & $\begin{array}{c}\text { Hirundinidae } \\
\text { Motacillidae } \\
\text { Pycnonotidae Laniidae } \\
\text { Turdinae } \\
\text { Timalinae } \\
\text { Estrilidae Passerinae Sturnidae } \\
\text { Dicruridae } \\
\text { Corvidae }\end{array}$ & 25 & 0 & 5 & 30 \\
\hline & 10 Orders & 37 Families & 53 & 37 & 13 & 103 \\
\hline
\end{tabular}

Table 3: Order, family and species as per the habitats.

used to had flocks of waders due to its shallow nature. The waterhen species (white-breasted waterhens, swamphens and moorhens) were the species found nesting within or near the waste water sites. Egrets and passerine birds were found nesting only when there was enough vegetation around waste dumping sites. The stretches of Ahar nallah were the observed sites for nesting along with those at Bharatpur. Thus, it was concluded that in presence of the natural resources and habitats, these human modified habitats could be one of the places attracting birds and could be used for birding.

\section{Discussion}

The avifaunal species richness is directly or indirectly affected by the environmental characteristics especially in the areas with the high rate of anthropogenic activities [14]. Surman [15] listed several factors of disturbances reflecting ecological and behavioral characteristics in birds. It is important to assess the micro habitats available to birds at a smaller spatial scale. As per the studies of Anon [16] anthropogenic factors are among the critically important factors in mapping the microhabitat site in terms of avifaunal sensitivity and ultimately informing the mitigation requirements. In the present investigation, it was observed that the study area harbors ninety percent of the avifauna in their habitats $[1,2,8,17,18]$. The species richness was greatly affected in the sites of disturbances $[1,2,8,17,18]$. The waste disposal sites (both solid and liquid) are exclusively observed under the present investigation. Tuljapurkar and Bhagwat [10] observed that the concentration of garbage at one place by civic authorities in towns and mega cities provides an ample supply of food to diverse species including birds. As expected the number of species was not up to the higher side as compared to the other sites low disturbances. Despite of the fact that the conditions were not favorable for the birds, the waste sites harbored substantial number of species. Since the present investigation was a short period investigation of a year and need long-term monitoring to 
Citation: Mehra SP, Mehra S, Uddin M, Verma V, Sharma H, et al. (2017) Waste as a Resource for Avifauna: Review and Survey of the Avifaunal Composition in and around Waste Dumping Sites and Sewage Water Collection Sites (India). Int J Waste Resour 7: 289. doi: 10.4172/22525211.1000289

recommend the sites as a useful resource for avifauna but it observed that the species which are resistant enough to the deteriorating environment use these sites for their life cycle processes. The life cycle processes are mainly food in terms of organic wastes $[19,20]$. This showed that the sites were not the primary choice of the observed species. If they get the better option, they might shift. Due to limited studies, such interpretations need justifiable study time period to conclude (Supplementyary Figures 1-3).

\section{Conclusion}

The dumping sites were mostly considered to be useful for a certain professionals of the community dealing with waste as wealth. But these sites could be of great utility for the raptorial species "Vulture Cafeteria" of global interest. The only need is to check and balance. The animal wastes and the biodegradable wastes could be used as a food resource for different species. Further, with the development of green belt around the dumping sites, one could attract the diversity of birds. Thus, these sites could be of great revenue generation for the local municipal bodies.

\section{References}

1. Mehra SP, Mehra S, Sen P (2013) Urban avifauna of Udaipur and its importance to the local population (Udaipur, Rajasthan, India). International Journal on Biodiversity Watch 2: 120-146.

2. Mehra SP, Mehra S, Sharma KK (2014) Importance of urban biodiversity - A case study of Udaipur, India.

3. Mehra S, Mehra SP, Sharma KK (2012) Importance of aquatic avifauna in southern Rajasthan, India. Daya Publishing House, Delhi.

4. Mehra SP, Mehra S (2017) Behavioral response of birds in stress with emphasis on globally important species Green Avadavat (Amandava formosa). Stress Management Professional An International Journal 5: 36-40.

5. Mehra SP, Mehra S (2014) Perspective on water and biodiversity issues: A case study of Keoladeo National Park, Bharatpur, India.

6. Wiens JA (1995) Habitat fragmentation: Island vs. landscape perspectives on bird conservation. Ibis 137: 97-104.
7. Andren $H$ (1994) Effects of habitat fragmentation on birds and mammals in landscapes with different proportions of suitable habitat: A review. Oikos 71 355-366.

8. Mehra S (2012) The avifauna of southern Rajasthan with special emphasis on threatened species and bioacoustic applications in their identifications and monitoring. Ph.D. Thesis, M. D. S. University, Rajasthan, India.

9. Annepu RK (2012) Sustainable solid waste management in India. MSc Dissertation, Columbia University, New York.

10. Tuljapurkar VB, Bhagwat $\mathrm{V}$ (2007) Avifauna of a waste disposal site. Indian Birds 3: 87-90.

11. Mehra SP (2006) Mount Abu: Step towards conservation.

12. Manakadan R, Pittie A (2001) Standarized common and scientific names of the birds of the Indian Subcontinent. Buceros 6: 1-37.

13. Kumar A, Satia JP, Tak PC (2003) Checklist of indian waterbirds. Buceros 8 1-30.

14. Oliveira LVL de (2013) Avifauna in areas with different stages of conservation in the Southern Espinhaço. Masters Dissertation, Federal University of the Jequitinhonha and Mucuri Valleys, Diamantina.

15. Surman CA (2006) Avifauna management plan for the long island tourism development, Wallabi Group, Houtman Abrolhos, Western Australia.

16. Anon (2015) Waste management license for silicon smelters. Draft Environmental Impact Assessment Report, Silicon Smelters (Pty) Ltd., WSP, Parsons Brinckerhoff, South Africa.

17. Mehra SP, Mehra S, Gautam P (2009) Avifaunal diversity of Keoladeo National Park: A review (Abstract). Wildlife Institute of India, Dehradun.

18. Mehra S, Mehra SP, Sharma KK (2011) Aquatic avifauan of Aravalli Hills Rajasthan, India.

19. Bird DM, Varland DE, Negro JJ (1996) Raptors in human landscapes: adaptations to built and cultivated environments. Academic Press, San Diego, CA.

20. Cringan AT, Horak GC (1989) Effects of urbanization on raptors in Western United States. 